\title{
Insights into structural and dynamical changes experienced by human RNase 6 upon ligand binding
}

\author{
Chitra Narayanan ${ }^{1, \dagger}$, David N. Bernard ${ }^{1}$, Myriam Létourneau ${ }^{1}$, Jacinthe Gagnon ${ }^{1, \&}$, Donald \\ Gagné $^{1, \$}$, Khushboo Bafna ${ }^{2, \#}$, Charles Calmettes ${ }^{1,3}$, Jean-François Couture ${ }^{4}$, Pratul K. Agarwal ${ }^{5, \$}$, \\ Nicolas Doucet ${ }^{1,3^{*}}$ \\ ${ }^{1}$ Centre Armand-Frappier Santé Biotechnologie, Institut National de la Recherche Scientifique (INRS), Université \\ du Québec, 531 Boulevard des Prairies, Laval, Quebec, H7V 1B7, Canada; ${ }^{2}$ Genome Science and Technology, \\ University of Tennessee, Knoxville, TN 37996, USA; ${ }^{3}$ PROTEO, the Québec Network for Research on Protein \\ Function, Engineering, and Applications, 1045 Avenue de la Médecine, Université Laval, Quebec, G1V 0A6, \\ Canada; ${ }^{4}$ Ottawa Institute of Systems Biology, Department of Biochemistry, Microbiology and Immunology, \\ University of Ottawa, 451 Smyth Road, Ottawa, Ontario K1H 8M5, Canada; ${ }^{5}$ Department of Biochemistry, Cellular \\ and Molecular Biology, University of Tennessee, Knoxville, TN 37996, USA; *Corresponding author: Email: \\ nicolas.doucet@inrs.ca
}

\section{Supporting Information}


Table S1. RNase residues involved in hydrogen bonding, van der Waals, and electrostatic interactions with a ligand bound to the $\mathrm{B}_{2}$ subsite, determined using the PDBeMotif server ${ }^{l}$. Residues displaying hydrogen bonding interactions with the phosphate groups are identified using an $\operatorname{asterisk}(*)$.

\begin{tabular}{|c|c|c|c|}
\hline RNase & Ligand & Hydrogen bonding & van der Waals interactions \\
\hline RNase 6 & 5'-AMP & Lys $7^{*}$ & $\begin{array}{l}\text { Cys62, Asn64, Arg66, Ala105, } \\
\text { D107, V121 }\end{array}$ \\
\hline RNase A & 5'-AMP & $\begin{array}{l}\text { Gln } 11^{*}, \text { Asn } 71 \text {, His } 119^{*} \text {, } \\
\text { Phe120* }\end{array}$ & $\begin{array}{l}\text { His12, Lys41, Cys65, Asn67, } \\
\text { Gln69, Ala109, Glu111, } \\
\text { Val118, His119 }\end{array}$ \\
\hline RNase 2 & $25^{\prime}-\mathrm{ADP}$ & $\begin{array}{l}\text { Gln } 14^{*}, \text { Asn } 70, \text { His } 129^{*} \text {, } \\
\text { Leu130* }\end{array}$ & $\begin{array}{l}\text { Met0, His } 15, \text { Cys62, Arg68, } \\
\text { Ala110 }\end{array}$ \\
\hline RNase 3 & $25^{\prime}-\mathrm{ADP}$ & Gln $14^{*}$, Lys $38^{*}$ & Met0, His15, Arg34, His 128 \\
\hline
\end{tabular}




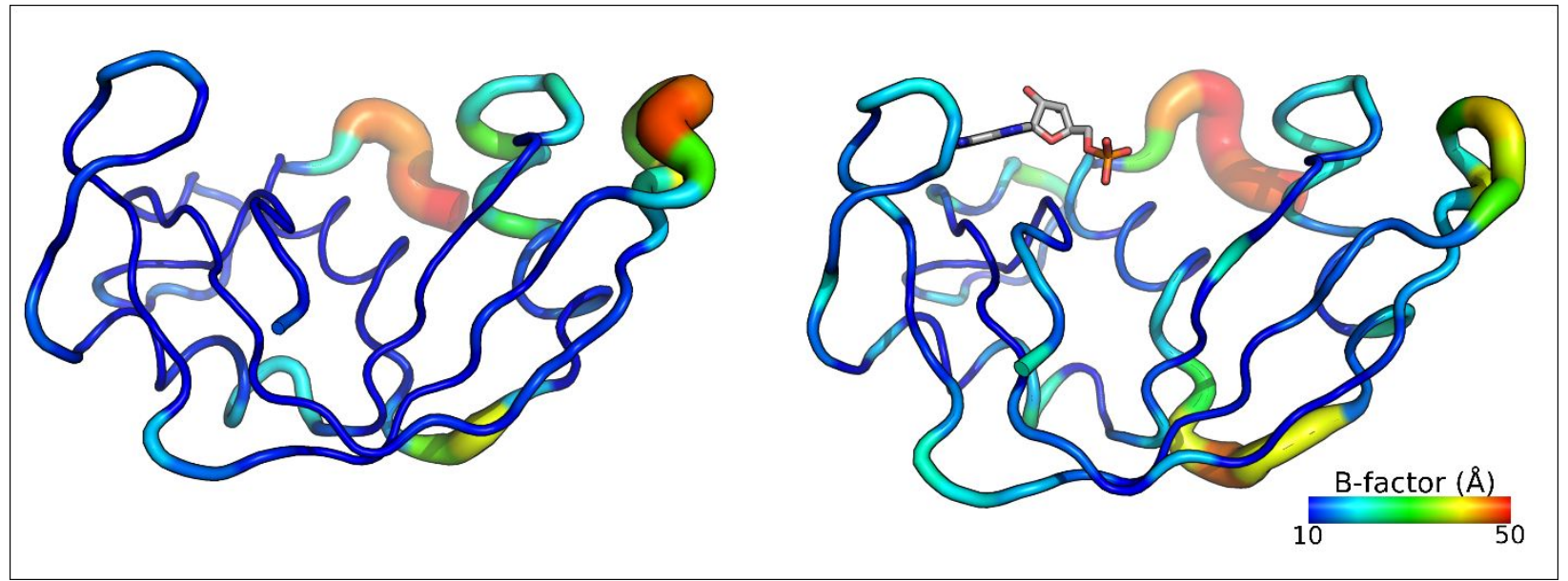

Figure S1. B-factor values determined from the crystal structures of RNase 6 in the apo (left) and 5'-AMP bound (right) states displayed using the putty representation. Thicker tubes with colors in the red end of the spectrum represent residues exhibiting larger B-factor values. 


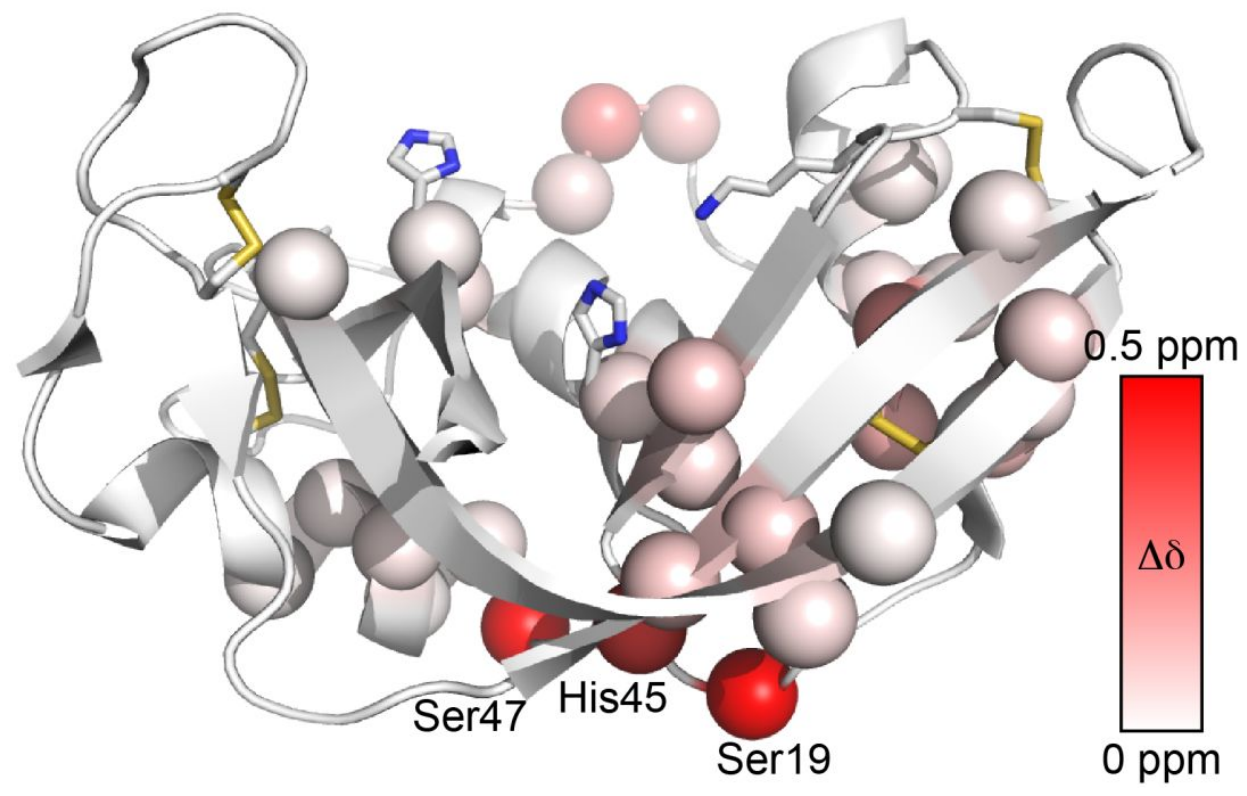

Figure S2. Location of duplicated NMR $\left\{{ }^{1} \mathrm{H}-{ }^{15} \mathrm{~N}\right\}-\mathrm{HSQC}$ resonance peaks mapped on the threedimensional structure of RNase 6. Residues in which at least one atom displaying two resonances in the $\left\{{ }^{1} \mathrm{H}-{ }^{15} \mathrm{~N}\right\}-\mathrm{HSQC}$ spectrum are depicted as spheres. The color gradient corresponds to the compounded chemical shift differences (ppm) between the two resonances on the $\left\{{ }^{1} \mathrm{H},{ }^{15} \mathrm{~N}\right\}-\mathrm{HSQC}$ spectrum. The three residues with the largest $(\sim 0.5 \mathrm{ppm})$ chemical shift differences are identified. The primary active-site residues (gray and blue) and the four disulfide bridges stabilizing the 3D structure (yellow) are depicted as sticks. 

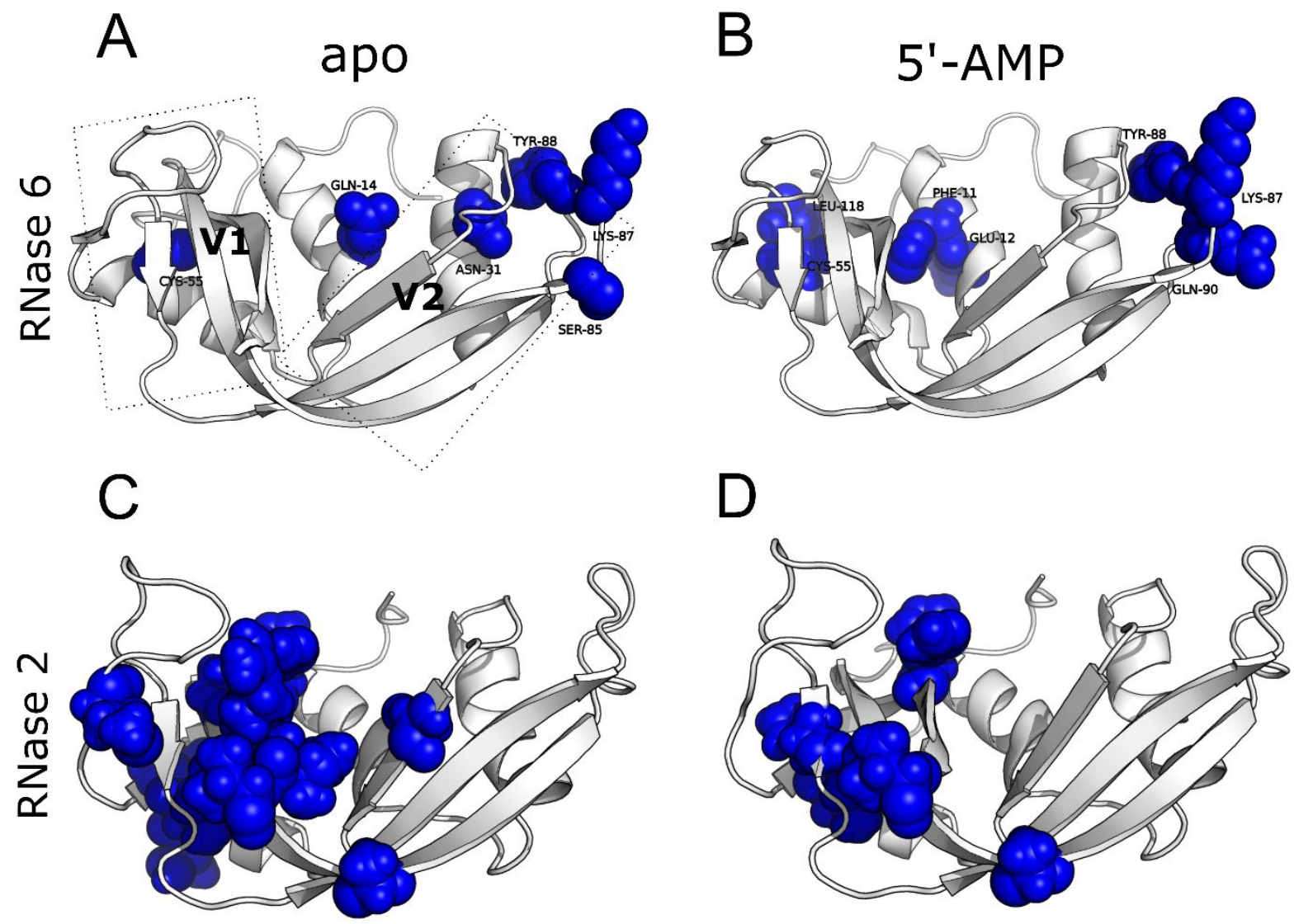

$\mathrm{D}$

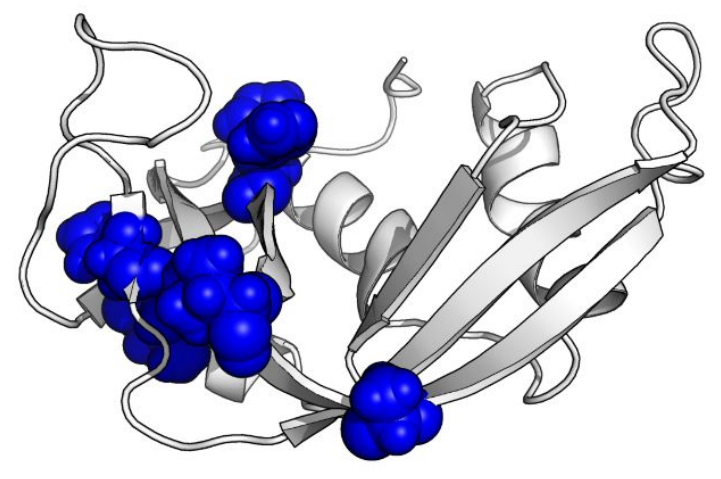

Figure S3. Millisecond timescale conformational exchange experienced by RNase 6 and RNase 2 in the free and 5'-AMP-bound states. ${ }^{15} \mathrm{~N}-\mathrm{CPMG}$ relaxation dispersion experiments were performed on the apo (A, C) and 5'-AMP-bound (B, D) states of the ${ }^{15} \mathrm{~N}$-labeled RNase 6 and RNase 2. Residues identified as undergoing conformational exchange on the millisecond timescale $\left(\Delta \mathrm{R}_{2, \text { eff }}(1 / \tau \mathrm{cp})>1.5 \mathrm{~s}^{-1}\right)$ are depicted using a space-filling representation. 


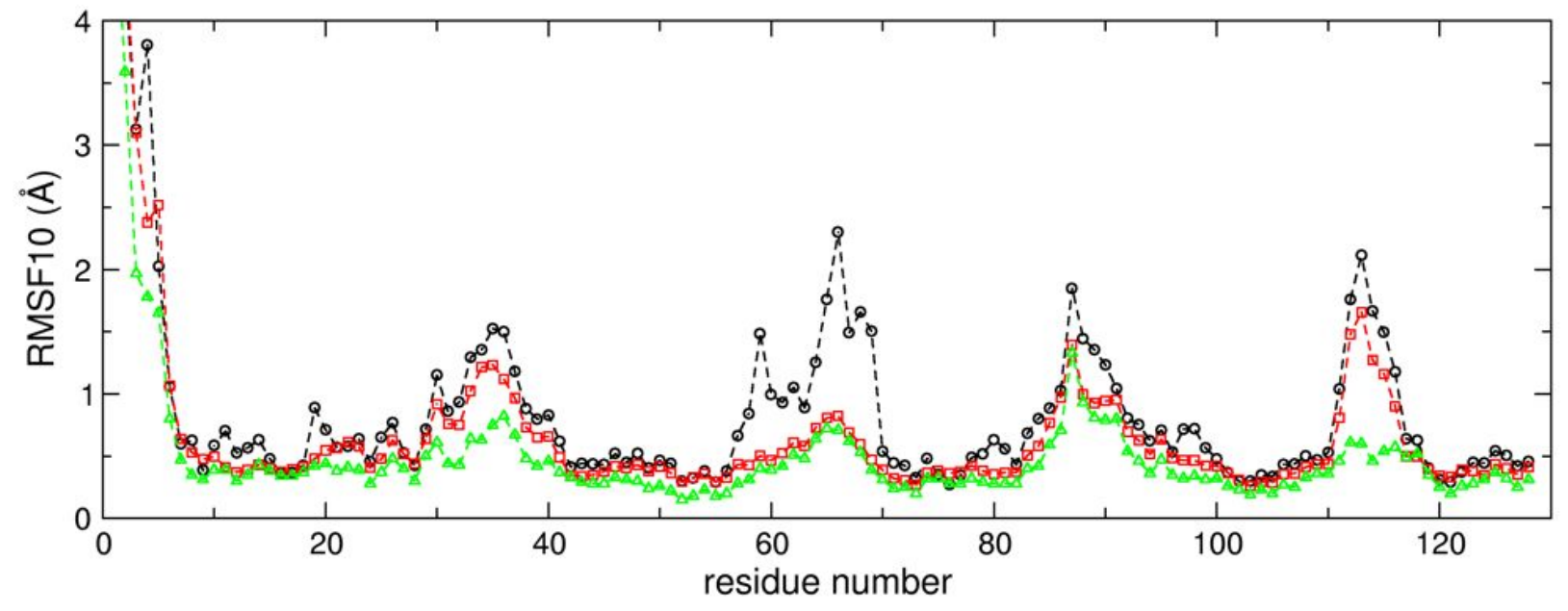

Figure S4. Root mean square fluctuations (RMSFs) of the top ten modes for the apo (black), 5'AMP- (red) and ACAC- (green) bound states of RNase 6. 

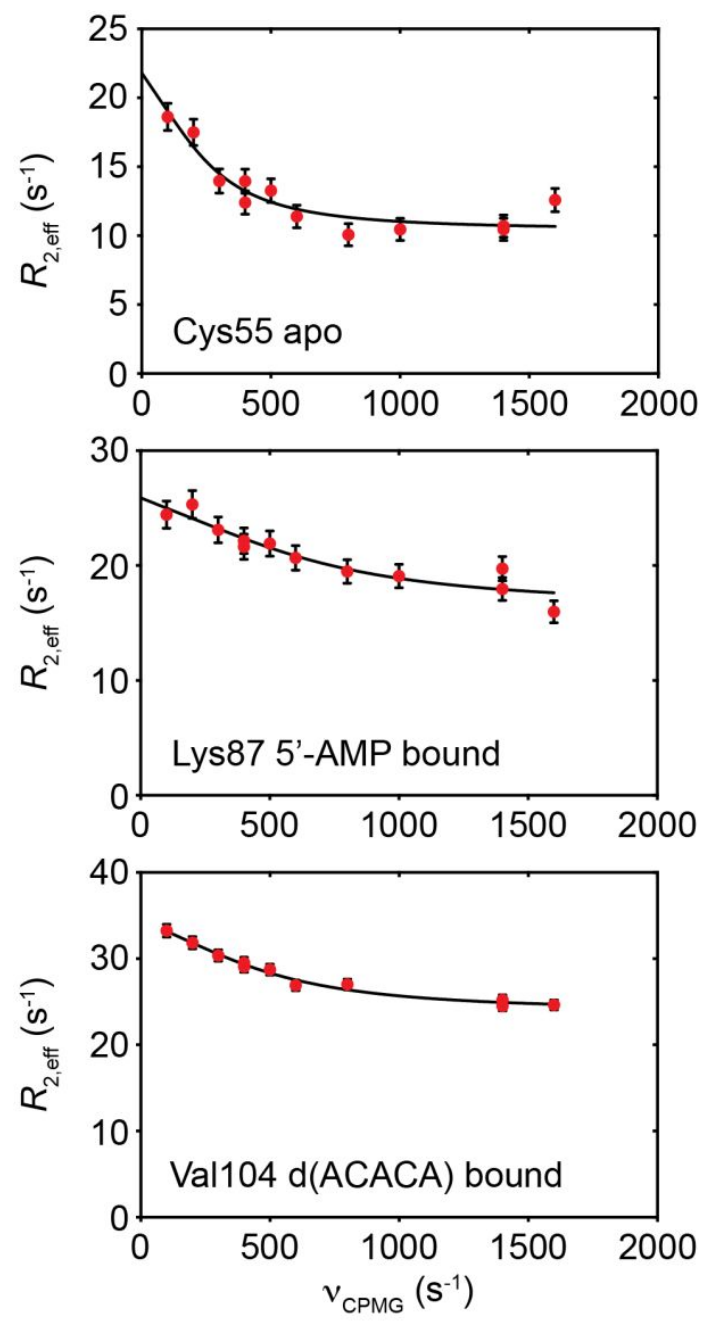

Figure S5. Representative ${ }^{15} \mathrm{~N}-\mathrm{CPMG}$ relaxation dispersion curves obtained for free and ligandbound RNase 6, measured at $800 \mathrm{MHz}$. Curves were fitted to the fast exchange relaxation dispersion equation ${ }^{2}$. 

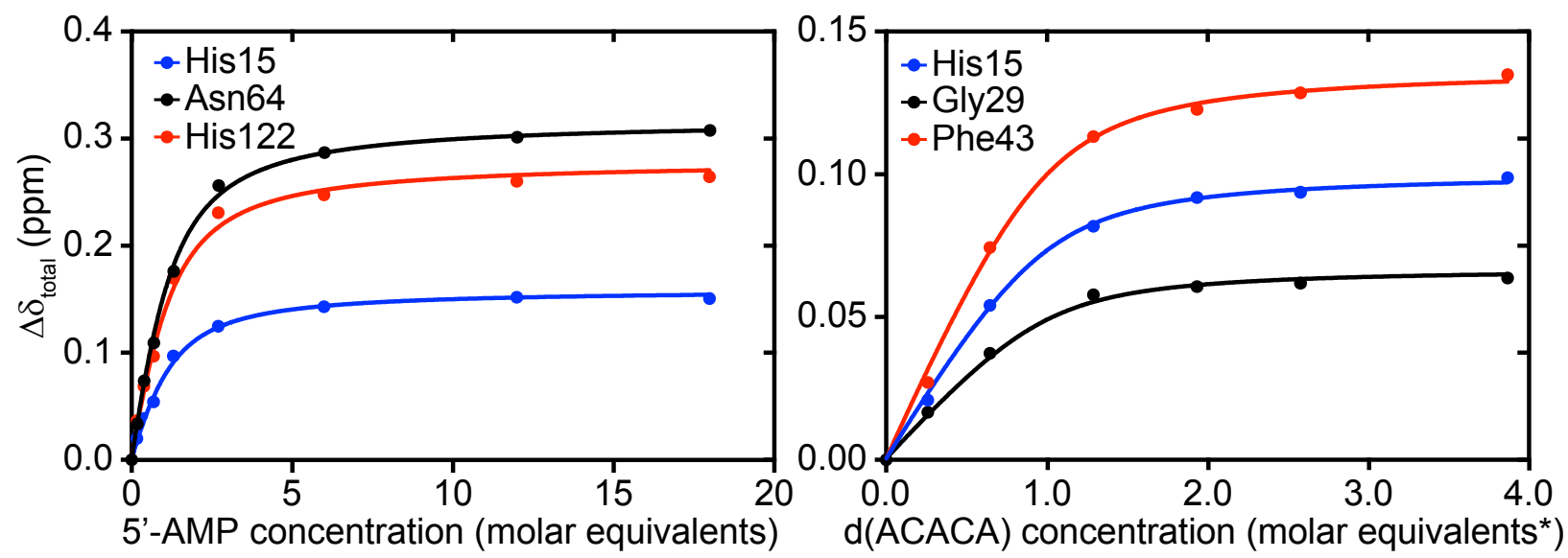

Figure S6. Representative NMR titration curves for both ligands, measured at $500 \mathrm{MHz}$. *The $\mathrm{d}(\mathrm{ACACA})$ concentration was scaled as described in the Methods section. 


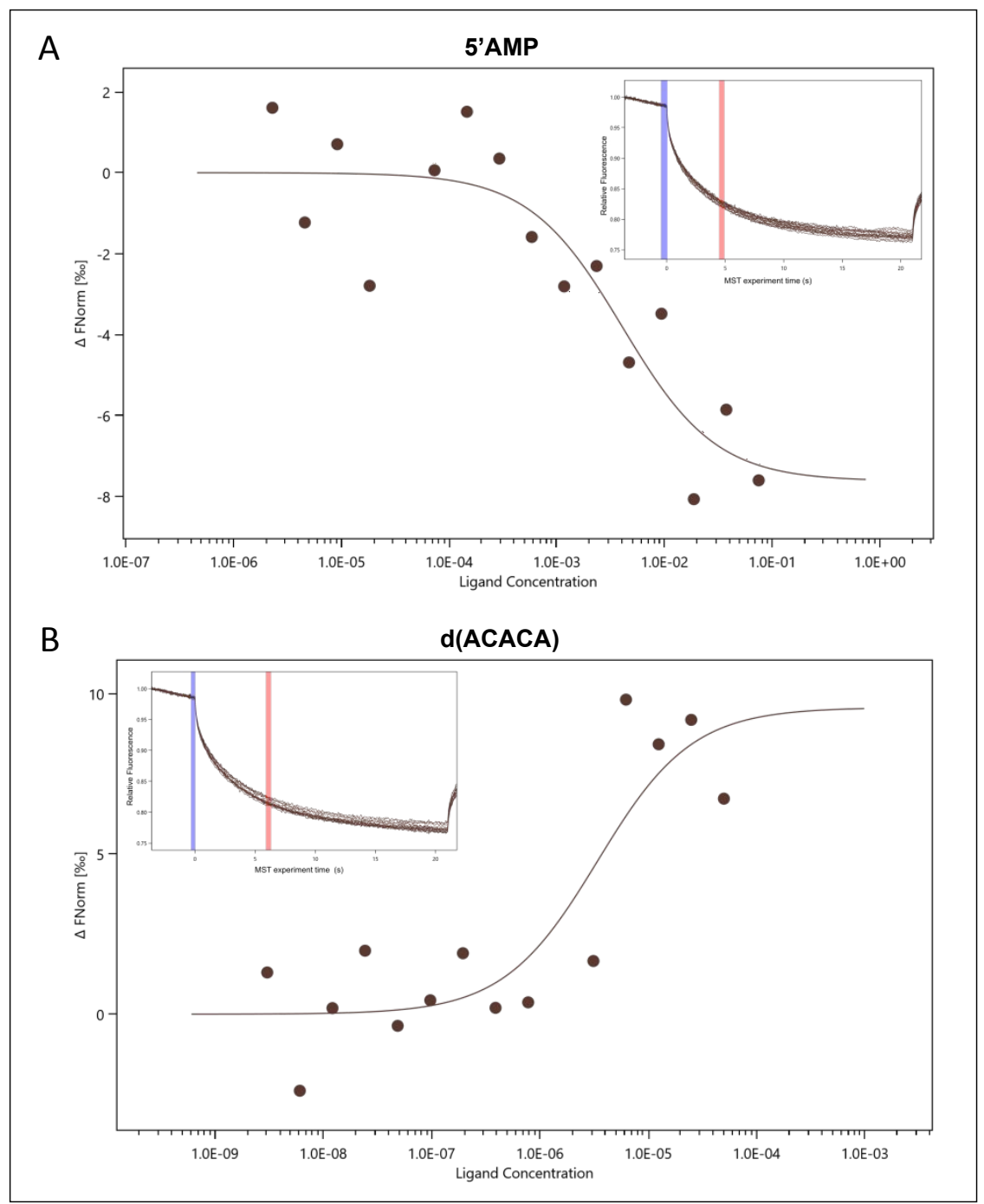

Figure S7. Representative microscale thermophoresis graphs obtained for baseline normalized fluorescence of labeled RNase 6 ( $\Delta$ Fnorm, in \%) plotted against ligand concentration. By definition, values start at 0 in the unbound state, adopting positive or negative values based on the ligand-dependent direction of the response. Normalized response of labeled RNase 6 (5 nM) was plotted against ligand concentration (M) to determine $K_{d}$ values in accordance with the law of mass action, using a 1:1 stoichiometry. MST traces are shown as insets, with baseline and MST-on time regions used to evaluate binding affinity highlighted in blue and pink, respectively. A) RNase 6 in presence of 5'AMP; B) RNase 6 in presence of d(ACACA). 


\section{References}

[1] Paxman, J. J., and Heras, B. (2017) Bioinformatics tools and resources for analyzing protein structures, Methods Mol Biol 1549, 209-220.

[2] Lisi, G. P., and Loria, J. P. (2016) Using NMR spectroscopy to elucidate the role of molecular motions in enzyme function, Prog Nucl Magn Reson Spectrosc 92-93, 1-17. 